\title{
Thoracic Epidural analgesia versus Rectus Sheath Catheters for open midline incisions in major abdominal surgery within an enhanced recovery programme (TERSC): study protocol for a randomised controlled trial
}

Kate M Wilkinson ${ }^{1}$, Anton Krige ${ }^{1 *}$, Sarah G Brearley ${ }^{2}$, Steven Lane ${ }^{3}$, Michael Scott ${ }^{4}$, Anthony C Gordon ${ }^{5}$ and Gordon L Carlson ${ }^{6}$

\begin{abstract}
Background: Thoracic epidural analgesia (TEA) is recommended for post-operative pain relief in patients undergoing major abdominal surgery via a midline incision. However, the effectiveness of TEA is variable with high failure rates reported post-operatively. Common side effects such as low blood pressure and motor block can reduce mobility and hinder recovery, and a number of rare but serious complications can also occur following their use.

Rectus sheath catheters (RSC) may provide a novel alternative approach to somatic analgesia without the associated adverse effects of TEA. The aim of this study is to compare the efficacy of both techniques in terms of pain relief, patient experience, post-operative functional recovery, safety and cost-effectiveness.

Methods/design: This is a single-centre randomised controlled non-blinded trial, which also includes a nested qualitative study. Over a two-year period, 132 patients undergoing major abdominal surgery via a midline incision will be randomised to receive either TEA or RSC for post-operative analgesia. The primary outcome measures pain scores on moving from a supine to a sitting position at 24 hours post wound closure, and the patient experience between groups evaluated through in-depth interviews. Secondary outcomes include pain scores at rest and on movement at other time points, opiate consumption, functional recovery, morbidity and cost-effectiveness.
\end{abstract}

Discussion: This will be the first randomised controlled trial comparing thoracic epidurals to ultrasound-guided rectus sheath catheters in adults undergoing elective midline laparotomy. The standardised care provided by an Enhanced Recovery Programme makes this a comparison between two complex pain packages and not simply two analgesic techniques, in order to ascertain if RSC is a viable alternative to TEA.

Trial registration: Current Controlled Trials ISRCTN81223298 (16 January 2014).

Keywords: Rectus sheath catheters, Rectus sheath block, Analgesia, Epidural, Enhanced recovery, Laparotomy

\footnotetext{
* Correspondence: anton.krige@elht.nhs.uk

'Department of Anaesthesia and Critical Care, Royal Blackburn Hospital, Blackburn BB2 3HH, UK

Full list of author information is available at the end of the article
} 


\section{Background}

\section{Background and rationale}

Enhanced Recovery Programmes (ERP) consist of a series of protocolised multimodal interventions aimed at reducing complications, thereby expediting recovery [1]. One of the key elements in all ERP is the provision of adequate post-operative analgesia. This reduces the stress response, improves patient wellbeing and allows early mobilisation. Early mobilisation itself is important to reduce secondary complications such as chest infection and deep vein thrombosis. Post-operative insulin resistance, which has been linked to increased complications, can be reduced by muscle activity [1]. Thoracic epidural analgesia (TEA), which facilitates dynamic analgesia and early mobilisation, is currently the standard for post-operative analgesia following major abdominal surgery within ERP [1].

A number of systematic reviews and meta-analyses have demonstrated that TEA is associated with superior postoperative analgesia and fewer adverse events when compared to high dose systemic opiate administration [2-8]. High dose systemic opiates are strongly associated with post-operative sedation, ileus [8-11] and nausea and vomiting, all of which impair post-operative recovery and prolong hospital stay. Ileus is the most common complication following abdominal surgery, and is responsible for significant prolongation of recovery times. TEA may reduce ileus rates as compared to opiates [12-17].

TEA is occasionally contraindicated and may also lead to rare but significant complications, for example nerve injury, epidural haematoma and epidural abscess [18]. More common adverse effects of TEA include motor blockade of the lower limbs and urinary retention requiring catheterisation, both of which can impede postoperative mobilisation and recovery. Additionally, the need to use epidural opioids to prevent the sensory regression associated with the use of local anaesthetics alone may contribute in itself to an increase in post-operative ileus [13-15]. Hypotension, resulting from sympathetic nervous blockade, is common with TEA and may result in iatrogenic fluid overload [19]. Fluid overload is also associated with ileus and other post-operative complications, including anastomotic dehiscence, and has been shown to independently predict increased length of hospital stay [20]. TEA has also been found to have a high reported failure rate (25 to $30 \%$ ) [2-8]. These failures are multifactorial and usually managed either by epidural replacement, substitution with systemic opiate infusion or both [21,22].

Rectus sheath catheters (RSC) are a regional anaesthetic technique in which the ventral rami of the seventh to twelfth intercostal nerves, which supply the rectus abdominis muscle and overlying skin, are blocked [23,24]. This provides midline somatic analgesia, with additional doses of systemic opiate required for visceral analgesia (12 to 36 hours duration) $[23,24]$. This sensory blockade is achieved by injecting local anaesthetic into the potential space between the rectus muscle and the posterior rectus sheath. As a single bolus of local anaesthetic has a maximum duration of 12 hours [23,24] it is necessary to insert a catheter into this space to allow either a continuous infusion of local anaesthetic, or repeated boluses of local anaesthetic every 8 to 12 hours for 48 to 72 hours post-operatively. Although this regional block was first described at the turn of the last century [23,24], it was used infrequently until the recent availability of long-acting local anaesthetic agents, small portable ultrasound machines [25] and small calibre infusion catheters. These developments have resulted in renewed interest in RSC for the management of post-operative pain following midline abdominal incisions [23,24,26-29].

RSC offer several potential advantages over TEA, which may make them ideally suited for use in an ERP. In particular, they avoid hypotension and motor blockade. RSC are also inserted after the induction of general anaesthesia, which patients may prefer to the usual awake insertion of epidurals. No complications or serious adverse events related to RSC have been reported to date [23,24], but local anaesthetic toxicity [30-33] (which applies to all regional anaesthetic techniques including epidurals) and visceral injury during insertion are hypothetical possibilities.

The existing literature relating to the use of RSC is limited, with published studies all utilising surgically inserted RSC to compare local anaesthetic with saline [34-40] or non-randomised trials comparing RSC to epidurals $[38,40]$. Despite this, the majority of studies demonstrate proof of concept [34-37,39]. This randomised controlled trial will compare ultrasound-guided insertion of RSC with TEA for midline laparotomy incisions, in adults undergoing elective major abdominal surgery within an ERP pathway.

\section{Objectives}

The aim of the study is to assess the efficacy, safety and acceptability to patients of RSC. To this end, a qualitative study has been nested within the primary study design to facilitate understanding of the patient experience.

\section{Trial design}

This is a randomised parallel group concealed allocation non-blinded superiority trial with a nested qualitative study of a subset of patients.

\section{Methods: participants, interventions and outcomes Study setting}

TERSC is a single-centre trial, which will take place at the Royal Blackburn Hospital ( $\mathrm{RBH})$, a large district general hospital in England providing services to a local 
catchment population of 530,000 and complex regional services to a population of 2 million.

\section{Eligibility criteria}

All adult (18 or over) patients who are listed for elective major abdominal surgery via a midline incision, and who meet all inclusion and exclusion criteria (see Table 1), will be eligible for recruitment into the trial.

\section{Interventions}

\section{Standard general anaesthetic}

All patients in the trial will receive a general anaesthetic as follows: propofol 2 to $4 \mathrm{mg} / \mathrm{kg}$ intravenously (IV); remifentanil $1 \mathrm{mcg} / \mathrm{kg}$ or target controlled infusion (effect site target-Minto model) or fentanyl 1 to $2 \mathrm{mcg} / \mathrm{kg} \mathrm{IV}$; atracurium $0.5 \mathrm{mg} / \mathrm{kg}$ or rocuronium $0.5 \mathrm{mg} / \mathrm{kg}$. Anaesthesia will be maintained as follows: isoflurane, sevoflurane or desflurane, initially at $1 \mathrm{MAC}$ (minimum alveolar concentration) then titrated to clinical end points; remifentanil (RSC arm only) 0.1 to $0.5 \mathrm{mcg} / \mathrm{kg} / \mathrm{min}$ or target controlled infusion (effect site target-Minto model) titrated to clinical effect; oxygen in air titrated to $\mathrm{SaO} 2>$ 95\%; and multimodal anti-emesis consisting of ondansetron 4 to $8 \mathrm{mg}$, dexamethasone $8 \mathrm{mg}$ and cyclizine $50 \mathrm{mg}$.

Maintenance fluid will consist of Plasma-Lyte B (Baxter, Compton, Newbury, Berkshire, UK) at 2 to $4 \mathrm{mls} / \mathrm{kg} / \mathrm{hr}$, and goal-directed fluid therapy utilising $250 \mathrm{ml}$ fluid

Table 1 Inclusion and exclusion criteria

\begin{tabular}{|c|c|}
\hline Inclusion criteria & Exclusion criteria \\
\hline - Patients $>18$ years of age & $\begin{array}{l}\text { - Contraindication to epidural } \\
\text { analgesia: for example, } \\
\text { coagulopathy, local infection, } \\
\text { systemic sepsis, severe aortic } \\
\text { stenosis }\end{array}$ \\
\hline $\begin{array}{l}\text { - Planned major abdominal } \\
\text { surgery including major } \\
\text { colorectal resections, } \\
\text { pancreaticoduodenectomy and } \\
\text { radical cystectomy }\end{array}$ & $\begin{array}{l}\text { - Consent refused for either TEA or } \\
\text { RSC }\end{array}$ \\
\hline $\begin{array}{l}\text { - Planned open midline surgical } \\
\text { incision }\end{array}$ & - Non-English speaker \\
\hline - Included in the ERP & $\begin{array}{l}\text { - Ano-rectal excision: for example, } \\
\text { pan-proctocolectomy or } \\
\text { abdomino-perineal resection. }\end{array}$ \\
\hline $\begin{array}{l}\text { - Willing and able to give } \\
\text { consent }\end{array}$ & $\begin{array}{l}\text { - Planned transverse or oblique } \\
\text { incisional approach }\end{array}$ \\
\hline \multirow[t]{4}{*}{$\begin{array}{l}\text { - ASA (American Society of } \\
\text { Anesthesiologists) } 1 \text { to } 3\end{array}$} & $\begin{array}{l}\text { - Allergy to local anaesthetic drugs or } \\
\text { opiates }\end{array}$ \\
\hline & - Opiate tolerance \\
\hline & - Pre-existing chronic abdominal pain \\
\hline & $\begin{array}{l}\text { - Extensive existing midline } \\
\text { abdominal scarring }\end{array}$ \\
\hline
\end{tabular}

Abbreviations: ERP, Enhanced Recovery Programme; RSC, rectus sheath catheter; TEA, thoracic epidural analgesia. boluses of Plasma-Lyte B until stroke volume is maximised. This will be guided by either oesophageal Doppler or Edwards Flotrac (Edwards Lifesciences, Irvin, California, USA) cardiac output monitoring.

\section{Rectus sheath catheters}

The RSC will be inserted bilaterally under ultrasound guidance (see Additional file 1 for description of the technique) [23-27] by the anaesthetist immediately following induction of a standard general anaesthetic. Only anaesthetists who have successfully inserted RSC under the supervision of the chief investigator (CI) will undertake the insertion of RSC. An aseptic technique will be used and $20 \mathrm{ml}$ of $0.25 \%$ bupivacaine will be injected via each catheter into the potential space between the rectus muscle and the posterior rectus sheath, 2 to $4 \mathrm{~cm}$ either side of the midline. The catheters will be tunnelled subcutaneously to a level above the costal margin.

Approximately 45 minutes before the end of surgery a 10-mg bolus of IV morphine will be administered to provide visceral analgesia. A transdermal fentanyl patch (12 mcg if $\geq 70$ years and/or $\leq 65 \mathrm{~kg}$ and $25 \mathrm{mcg}$ if $<70$ years and $>65 \mathrm{~kg}$; 72-hour duration of action) will be applied, and if more than 3 hours have passed since the initial RSC bolus, a further $40 \mathrm{ml}$ of $0.2 \%$ ropivacaine will be administered via an $\operatorname{ambIT}^{\mathrm{R}}$ (Summit Medical Products Inc, South Sandy, Utah, USA) Preset PCA or ambIT $^{\mathrm{R}}$ mini infusion pump (split between the 2 RSC via a Y-connector). The pumps will be set to deliver boluses of $40 \mathrm{mls} 0.2 \%$ ropivacaine, with a 4-hour lockout, each time the bolus button is pressed [30-33]. The bolus button may be staff- or patient-activated but will be pressed regularly every 4 hours post-operatively. Each $40 \mathrm{ml}$ bolus takes 24 minutes to deliver; therefore the total time from the start of one bolus to the start of the next will be 4 hours and 24 minutes.

If breakthrough pain is experienced, staff will first ensure that the RSC have been utilised as per protocol. If more than 4 hours have elapsed since the last bolus of local anaesthetic then $40 \mathrm{mls}$ of $0.2 \%$ ropivacaine will be administered via the pump. If inadequate pain relief follows the bolus, or if no bolus is due, then $10 \mathrm{mg}$ oral morphine or $5 \mathrm{mg}$ oral oxycodone will be used as required for further breakthrough pain within the first 48 hours.

\section{Thoracic epidural analgesia}

Epidurals will be sited prior to the induction of general anaesthesia at T7 to T9 for a right-sided colonic resection or upper gastrointestinal (GI) surgery, and T9 to T11 for a left-sided resection or radical cystectomy. Standard aseptic insertion technique will be followed, using loss of resistance to air or saline as per preference. Following a 
suitable test dose, a bolus of $10 \mathrm{mls} 0.25 \%$ bupivacaine with 100 mcg fentanyl will be administered to establish a block.

Following insertion, an epidural infusion of $0.125 \%$ bupivacaine and $2 \mathrm{mcg} / \mathrm{ml}$ fentanyl will be commenced at $10 \mathrm{mls} /$ hour and then titrated to effect. On the second post-operative night, a fentanyl patch $(12 \mathrm{mcg}$ if $\geq 70$ years and/or $\leq 65 \mathrm{~kg}$ and $25 \mathrm{mcg}$ if $<70$ years and $>65 \mathrm{~kg}$ ) will be applied after which the epidural will be weaned overnight and removed the following morning as per local clinical protocol.

If breakthrough pain is experienced, the epidural block will first be optimised with an additional bolus of 10 to $20 \mathrm{mls}$ of the maintenance solution. If inadequate pain relief follows the bolus then $10 \mathrm{mg}$ oral morphine or $5 \mathrm{mg}$ oral oxycodone will be used as required for further breakthrough pain within the first 48 hours.

All reasonable attempts will be made to rescue failing study interventions. These may include delivering an additional bolus of local anaesthetic, altering the catheter position, correcting blood pressure to allow continuation of the study intervention, and (in the case of TEA) repositioning the patient. However, if complete failure of the study intervention occurs within the first 48 hours postoperatively, it will be replaced by IV morphine patient controlled analgesia (PCA) following an adequate IV bolus of morphine. Complete failure is defined as complete lack of any sensory blockade and any improvement in the severity of pain reported by the patient following an adequate bolus injection of local anaesthetic via the TEA or RSC.

Patients in both arms of the trial will receive all other hospital care according to our institution's ERP. Preoperatively, this consists of pre-operative assessment, planning and preparation (including cardiopulmonary exercise testing if aged $\geq 60$ years or $<60$ years with cardiorespiratory comorbidity), ERP information, a patient diary and PreLoad (Vitaflo International Limited, Liverpool, UK) drinks (sachets of maltodextrin powder for reconstitution in $500 \mathrm{mls}$ water; 2 to be consumed the night before and an additional one 2 to 3 hours prior to surgery). Upper GI and right-sided colonic resections receive no bowel preparation and left-sided colonic resections, rectal resections and radical cystectomies will receive phosphate enemas on the morning of surgery. All patients receive $1 \mathrm{~g}$ paracetamol and $300 \mathrm{mg}$ gabapentin orally pre-operatively.

Intra-operatively, in addition to the standard general anaesthetic and interventions already described, nasogastric tubes are avoided, surgical drains minimised and normothermia is maintained using enFlow ${ }^{\circ}$ (GE Healthcare, Little Chalford, Buckinghamshire, UK) IV fluid warmers and Bair Hugger ${ }^{\text {rm }}$ (3 M, St Paul, Minnesota, USA) external warming blankets.
Post-operatively, in addition to the randomised primary analgesia technique all patients receive multimodal oral analgesia, comprising $1 \mathrm{~g}$ paracetamol 6-hourly orally or IV if not tolerating oral; $400 \mathrm{mg}$ ibuprofen 8-hourly orally once tolerating full oral diet; and $100 \mathrm{mg}$ gabapentin 8-hourly orally (for 72 hours). A transdermal fentanyl patch is applied with dose and timing relevant to the randomised intervention as described above. Post-operative nausea and vomiting is minimised with $3 \mathrm{mg}$ Buccastem 8-hourly buccally for 48 hours. Patients will undergo structured mobilisation from day 1 post-operatively. IV fluids are minimised and 2 litres per day of oral fluid is encouraged with full oral diet encouraged from day 1 postoperatively. Urinary catheters will be removed on day 1 in the RSC group and on removal of the epidural in the TEA group.

\section{Qualitative study}

A cross-sectional study will achieve the aim to study the patient experience of both interventions. This will utilise an inductive approach informed by the principles of grounded theory [41]. It uses maximum variety sampling to enable a range of experiences to be sought rather than seeking to make generalisations based on population characteristics. The anticipated sample size is 10 per trial arm (20 in total) and interviews will be undertaken by an experienced researcher at a place convenient to the participant (usually their home). During the qualitative phase all consenting RCT trial participants will be invited to take part in the qualitative study RCT (randomised controlled trial). They will be contacted again 2 to 3 weeks after the intervention, invited to participate and re-consented to avoid any issue of coercion whereby patients feel that they have to consent to the qualitative study in order to gain entry onto the RCT.

Face-to-face interviews will then take place 4 weeks post-intervention. This time point has been chosen to avoid the initial recovery period after hospital discharge, whilst still allowing the generation of rich data concerning the experience of RSC or TEA (we expect most patients to be discharged between 8 and 14 days, and to require a 3-month leave of absence from work). Data will be collected through in-depth interviews focusing on the participant's expectations, experiences and outcomes. The interviews will be digitally audio recorded and fully transcribed (specifically covered in the consent process). Constant comparative thematic analysis will be undertaken to detect commonalities and differences within the accounts and across the two groups. Constant comparison permits the early stages of analysis to identify emergent issues and inform later data collection in order to produce insights grounded in the experiences of the participants. 


\section{Outcomes}

The primary outcome measure of the RCT is the difference in mean pain score on movement from supine to sitting position at 24 hours after extubation between groups, measured using a Visual Analogue Scale (VAS) of 0 to $100 \mathrm{~mm}$ with 0 being no pain and $100 \mathrm{~mm}$ being the worst pain imaginable $[42,43]$. VAS is a standard tool to compare analgesic efficacy between interventions in pain studies, and pain control adequate to allow dynamic movement at 24 hours is considered key to successful recovery after major surgery, with full mobilisation thus potentially feasible. An additional primary outcome measure is the difference in patient experience measured by the nested qualitative study utilising interviews conducted one month after surgery in a subset of patients from the main trial.

Secondary outcomes include further comparisons of analgesic efficacy and effectiveness, functional recovery, safety and cost-effectiveness. Further analgesic quality measurements include mean VAS pain scores at rest and on movement at 2, 6, 12, 24, 48 and 72 hours after extubation between intervention groups to compare the efficacy of analgesia at different clinically relevant time points and the time-based evolution of analgesic efficacy; the difference in sleep quality measured by VAS scores on the first three mornings post-operatively; the difference in self-assessed functional pain categorising respiratory function and mobility measured between $4 \mathrm{pm}$ and $5 \mathrm{pm}$ on each of the first 3 post-operative days; time to first rescue opiate; the total opiate consumption (excluding the protocolised fentanyl) over the first 48 hours post-operatively; and an overall score of satisfaction with pain relief on day 3 post-operatively.

Functional recovery will be compared assessing the difference in decrement of the Post-operative Quality Recovery Scale (PQRS) score [44] between groups, from baseline measurement to the measurements obtained on days 4,7 and 30 post-operatively using a validated questionnaire [44]; time to first mobilisation; the difference in time to return of GI function; achievement of the daily ERP mobilisation goals (for example, $4 \times 60 \mathrm{~m}$ walks on day 2 post-operatively); adherence to the local ERP pathway; time until patients meet standardised clinical discharge criteria as per the hospital ERP [45,46]; and the actual length of hospital stay. Return of GI function is defined as the time to tolerance of full diet, passage of flatus and bowel opening. Tolerance of a full diet will occur when the patient has consumed more than $90 \%$ of the planned intake of a meal, and does not feel nauseated or vomit after any meal. If four meals are consumed and the fourth vomited, then time to tolerating a full meal will not have been reached.

Safety measurements are differences in fluid balance and the incidence of hypotension over the first 48 hours post-operatively. Overall fluid balance will be measured using the fluid balance charts and the difference between pre-operative body weight and body weight measured on the first three mornings post-operatively (positive fluid balance is strongly correlated with morbidity in this cohort and may be linked to side effects of the trial interventions, that is hypotension). Any episode of hypotension, defined as a systolic blood pressure less than $90 \mathrm{mmHg}$, a mean arterial pressure less than $65 \mathrm{mmHg}$, or a reduction of greater than $20 \%$ from the pre-operative blood pressure, will be noted. Vasoconstrictor usage will be measured as a surrogate of haemodynamic stability.

Other measures of morbidity include nausea and vomiting measured by a VAS score, the incidence of ileus and the POMS (Post-Operative Morbidity Score). Ileus has been defined as new nausea and vomiting, abdominal distension, and abdominal discomfort with loss of bowel sounds [47]. The POMS is a score of post-operative morbidity validated in the UK which applies a binary outcome to each of 9 domains on either day 3, 5 or 7 post-operatively, chosen depending on the type of surgery [48]. Day 5 will be used for this study. Any surgical complications occurring within 30 days postoperatively will be classified for severity using the ClavienDindo classification [49].

If a study intervention is deemed to fail, then the timing of the failure, any replacement technique and the reason will be recorded: for example, block failure (pain and no demonstrable block of abdominal dermatomes); catheter disconnection; excessive catheter leakage; premature catheter removal; cessation of infusion due to hypotension; respiratory depression or possible local anaesthetic toxicity (respiratory rate $<8 /$ minute or the need for naloxone, systolic $\mathrm{BP}<80$ or $\mathrm{MAP}<50$; pruritus; circumoral paraesthesia; seizures; obtundation; arrhythmias; and cardiovascular collapse).

A full health economic assessment calculating Quality Adjusted Life Years is beyond the scope of this study. However, short-term cost-effectiveness will be calculated, taking into account the cost of consumables, insertion and management time for the 2 techniques, the respective length of stay both in hospital and critical care, and readmissions or re-operations within 30 days of surgery [50].

\section{Participant timelines}

When patients are informed of the need for an operation by their surgical team, verbal agreement will be ascertained for their details to be forwarded to the trial team, enabling the trial nurse to approach them with verbal and written information (see Trial Schema, Figure 1). If willing, a verbal discussion can take place that day, otherwise patients will be given a copy of the Patient Information sheet and then contacted by the trial nurse to discuss the trial a few days later. Most patients will attend the 


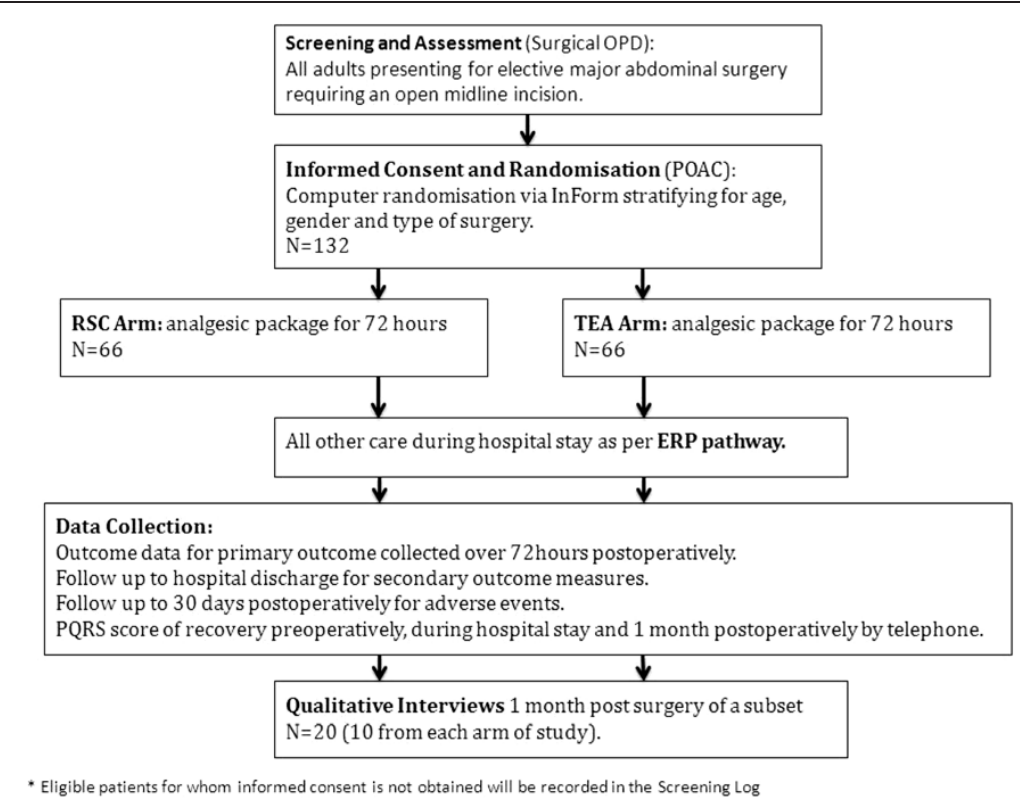

Figure 1 Trial schema. Diagram illustrating timelines and patient pathways for both study arms.

Pre-Operative Assessment Clinic (POAC) within 2 weeks of the decision to operate, and the trial nurse will meet them there to answer any further questions. If willing to participate, they will be consented and randomised, following baseline data collection, at that visit. Randomisation at this point will enable workforce planning for the day of surgery, as well as participant education on the ERP and trial data collection. However, some patients may be initially identified and approached at the POAC stage, and may therefore be randomised up to and including the day of admission.

The trial interventions will take place in the anaesthetic room on the day of surgery, with intensive data collection up to the third day post-operatively, and further specific data collection up to 30 days post-operatively.

\section{Sample size}

The primary end point is difference in VAS pain scores on movement at 24 hours post-operatively. The Kelly study [51] showed that the minimum clinically significant VAS pain score when managing severe pain was $10 \mathrm{~mm}$. Standard deviations (SD) varying from $14 \mathrm{~mm}$ to $18 \mathrm{~mm}$ have been reported [3-7], thus we have estimated a standard deviation of $18 \mathrm{~mm}$ for our study. To achieve $85 \%$ power to detect a $10 \mathrm{~mm}$ difference in the primary end point, from a VAS pain score on movement at 24 hours of $40 \mathrm{~mm}(\mathrm{SD}=18 \mathrm{~mm})$ in the TEA group, to $30 \mathrm{~mm}(\mathrm{SD}=18 \mathrm{~mm})$ in the RSC group, at the $5 \%$ level (two-sided $t$-test), will require 60 patients in each arm of the study.

An additional 6 subjects will be recruited in each study arm to cover a maximum of $10 \%$ losses (the MASTERS study [4] had only $3.5 \%$ losses), bringing the total sample to 132 subjects recruited over 24 months. This sample size is realistic as we have a recruitment pool of 130 admissions per year and expect $70 \%$ to be both eligible and willing to participate.

\section{Recruitment}

Relevant surgical teams, and in particular the specialist nurses, have been educated on trial eligibility criteria and will communicate clinic dates for potential participants to the trial nurse once a decision to operate is made. Most of these decisions take place at the weekly Multidisciplinary Cancer Meeting, and the trial nurse will therefore attend the colorectal meetings.

In addition, all relevant surgical outpatient clinics have been provided with eligibility posters and trial nurse contact details as further reminders. POAC personnel have also received this material, as have the admissions clerks who are responsible for booking patients into outpatient and operation dates. This will identify any additional patients who may have been missed initially. Clinic dates for benign disease will similarly be communicated to researchers (this only applies to colorectal surgery) for all potentially eligible patients.

The trial nurse will then attend the relevant surgical clinic where patients will be offered major surgery (or POAC if identified at this stage). Eligible patients will be asked by the surgical team if they would be willing to be approached about the study by the research team, or have their contact details forwarded to the research team. If they agree, the research nurse will be on hand for an initial meeting where a verbal explanation of the study will 
be provided, along with a copy of the Patient Information Sheet.

Some patients may not feel ready to have a further research related discussion on the same day, but still be open to the idea of participation. The research nurse will gain their permission to contact them in a few days to discuss the study, and they will be provided with the Patient Information Sheet and contact details. As part of their surgical pathway, patients will be given an appointment at the POAC within 2 weeks. The research nurse will meet them again at this clinic and ascertain if they wish to participate: if so, they will be randomised, following consent and collection of baseline data. Patients who are approached for the first time at the POAC may have this second discussion ( \pm randomization) on the day of admission, allowing time for consideration.

Only patients with mental capacity and the ability to consent will be recruited. Patients who are eligible but not randomised, and those who fulfil the inclusion criteria but meet one or more of the exclusion criteria, will be recorded in the TERSC Screening Log, irrespective of whether consent is obtained.

All stakeholders, that is anaesthetists, surgeons and specialist nurses, will be updated regularly of recruitment to the trial to ensure continued support and encouragement with positive feedback. The monthly Trial Management Group and the 6-monthly Trial Steering Committee (TSC) will scrutinise the recruitment rate and provide further suggestions and recommendations to increase any failing recruitment.

\section{Methods: assignment of interventions}

\section{Allocation: sequence generation}

Patients will be stratified by operation type and age, with three surgical strata (radical cystectomies, major colonic excisions and major rectal excisions) and two age strata (up to and above forty years of age) by computer generated random numbers. Of the 66 patients recruited into each arm of the study, we expect $50 \%$ to be major colonic excisions, $30 \%$ to be major rectal excisions and $20 \%$ to be radical cystectomy cases. Because we expect to see substantially more patients in the over-40 group, a 2:1 inclusion rate between the age groups is expected. It should be noted that the radical cystectomy group will only contain patients over the age of forty, and, therefore, in total there will only be five strata. The Trial Statistician has provided the InForm database designers with lists of the random assignment blocks, without any involvement of the $\mathrm{CI}$ or trial nurse.

\section{Allocation: concealment mechanism}

The allocation is provided electronically by the InForm system as described above and is thus concealed to the research team.

\section{Implementation}

The trial nurse will enrol patients, and once eligibility criteria, operation type and demographics are entered, the InForm system will electronically randomise participants and assign the interventions.

\section{Blinding}

Only the data analysts (trial statistician) will be blinded to the assigned intervention when assessing the primary outcome measure.

\section{Methods: data collection, management and analysis}

\section{Data collection methods}

The trial research nurse is responsible for recruitment and data collection and has been trained by the CI. This training included the correct collection of all trial data along with definitions of data items and clinical scoring tools, correct assessment of pain intensity and other VAS as well as the PQRS score.

Baseline data is obtained in the POAC directly from the patient and the case notes. All outcome data is either obtained from the Patient Diaries, case notes, prescription, fluid balance and observation charts, or directly by the research nurse: for example, timing of intervention insertion, duration of surgery, length of wound, presence and location of drains and stomas, the use of cardiac output monitoring to optimise fluid management and haemodynamic data from monitors.

Patients are educated in the completion of the Patient Diaries by the research nurse at the pre-operative assessment visit. The research nurse and clinical staff then prompt patients to self-complete the diaries at specified time points throughout their stay. These diaries capture the outcome measures regarding pain control, nausea, sleep quality, gut function and fitness for discharge.

There are no paper Case Report Forms and all data collected by researchers will be entered directly onto a trial-specific eCRF (electronic Case Report Form) on the InForm database. This system promotes data quality by preventing duplicate data entry, generating queries whenever data deviates from set ranges, and requiring completion of mandatory fields as defined by the CI. An audit trail is generated for any data queries, which must be resolved before sign-off of completed data by the CI.

A local Research and Development Department quality manager will audit the quality of data collection periodically and the Data Management and Ethics Committee (DMEC) will forward reports of data quality from InForm to the TSC. Full data collection will continue for all patients that deviate from the protocol, and patients who discontinue in the trial will still be assessed for surgical complications, hospital length of stay and adverse events up to 30 days. 
Several validated scoring systems are used for predicting risk and for various outcome metrics. Peri-operative risk will be measured using the ASA (American Society of Anesthesiologists) score [52], and the Physiologic and Operative Severity Score for the enUmeration of Mortality and morbidity (p-POSSUM) score (a validated general surgical risk prediction score) [53]. Quality of Recovery will be assessed using the PQRS. This is a rapidly conducted instrument allowing assessment of several aspects of recovery over time. Physiological, nociceptive, emotional and cognitive domains are assessed, with a baseline (pre-operative) measurement followed by measurements over several time points. A feasibility study demonstrated high face validity with applicability to a wide age range, diverse languages, cultures, and physical abilities [44].

Details regarding the research interventions, for example, insertion, maintenance, protocol violations, adverse events, compliance with protocolised adjuvant analgesia and the ERP pathway, and all additional opiate analgesia will be obtained from case notes or directly by the research nurse. The hospital electronic administration system will be interrogated at 30 days for any readmissions to hospital, ensuring full capture of any adverse events in this time. The patients will be contacted at 30 days to obtain a final PQRS recovery score by telephone, and this will be deemed the endpoint for the primary study. A subset of patients will go on to be interviewed for the nested qualitative study.

\section{Data management}

All patient trial data will be entered directly into the secure web-based InForm database eCRF using a tablet device. Patient diaries, PQRS paper questionnaires and the medical notes will serve as source data. The CI will be responsible for data collection, quality and recording, however the collection of data will be delegated to the trial research nurse. This has been recorded in the Delegation of Trial Duties Log and authorised by the CI.

All data entered into the eCRF will be anonymised with only initials and date of birth entered. A separate and secure log will be kept with consent forms and identifiers to allow patient follow-up to 30 days. This is detailed in the Patient Information Sheet and emphasised on the consent form. During the conduct of the trial, all electronic patient data will be encrypted and all trial documents stored securely. On completion of the trial, all patient data (electronic and paper) and other trial documents will be archived securely and retained for 5 years at $\mathrm{RBH}$ in the case of paper documents and Imperial Clinical Trials Unit (CTU) in the case of electronic data.

Imperial CTU is registered under the Data Protection Act 1998 and all Imperial CTU staff have undergone data protection and International Conference on
Harmonisation (ICH) Good Clinical Practice (GCP) training. The $\mathrm{CI}$ and all research staff at the Trial Centre have undergone GCP training.

The InForm database generates automatic alerts for missing data, invalid data, data duplication or data not conforming to the rules established for that data type. Thus, validation is ongoing throughout the data entry process. Final data validation occurs at Imperial CTU. This will ensure all data is complete, accurate and consistent. The trial nurse will resolve data queries generated by the database as soon as possible.

TERSC will be managed according to the Medical Research Council's (MRC) Guidelines for Good Research Practice, Guidelines for Good Clinical Practice (GCP) in Clinical Trials, and Procedure for Inquiring into Allegations of Scientific Misconduct.

\section{Statistical methods}

A full statistical analysis plan will be written, a priori, before the investigators are unblinded to any trial outcomes by the trial statistician. All analyses will be performed according to the intention-to-treat principle. An additional per protocol analysis will be performed and reasons for any protocol violations reported. Baseline covariates will be reported for the two groups and described using summary statistics. To determine if the two groups are balanced, standard hypothesis tests ( $t$-test, MannWhitney $U$-test and Chi-squared test) will be applied. Prior to writing the statistical analysis plan, we will determine if the analyses of the primary or secondary outcomes need to be adjusted for significant clinical or demographic covariates. However, we will only adjust the analysis if significant differences in these covariates exist between the treatment and control groups. The primary analysis will test the null hypothesis that there is no difference in mean VAS pain scores on movement at 24 hours postoperatively between those receiving TEA and RSC analgesia. The independent sample $t$-test or Mann-Whitney $U$-test will be used to assess between group differences unless the preliminary analysis indicates a significant difference in the clinical or demographic covariates thought to have a significant impact on the primary outcome. If so, then analysis of covariance will be used to assess the primary outcome. As there are six follow-up measurements, a repeated measures analysis of variance will also be used to assess differences between groups, and over time.

The secondary outcomes will be analysed in a similar manner to the primary outcomes. Time to event variables, such as time to recovery of gut function and length of hospital stay will be analysed using Mann-Whitney $U$-test, log-rank or Cox regression if censoring occurs. To avoid missing data we will monitor the data on a regular basis, monthly, and when possible return to the source of the missing data to determine if data is available. At 
database lock, we will assess the nature of any missing data and use appropriate imputation methods to estimate missing values.

\section{Methods: monitoring Data monitoring}

The DMEC will consist of an independent academic clinician and an independent statistician, and will meet every 6 months. Operating under the DAMOCLES Charter $[54,55]$, the DMEC will receive reports on recruitment rates, adverse events, completeness of data collection and protocol violations, and will report in turn to the TSC.

\section{Harms}

Adverse events $(\mathrm{AE})$ and serious adverse events $(\mathrm{SAE})$ are defined in this trial according to Directive 2001/20/ EC, 4 April 2001, of the European Parliament (Clinical Trials Directive) and ICH (International Conference on Harmonisation) GCP E6 guidelines. All AE occurring in the 30-day follow-up period will be recorded and evaluated for severity and causality. Severity will be graded 1 (mild) through 5 (fatal) and causality will be graded as either none, unlikely, possible, probable or definitely related to the intervention. SAE will also be evaluated for expectedness. SAE will be reported to the Chief Investigator $(\mathrm{CI})$ within 24 hours. The CI will assess all SAE to determine the need for expedited reporting to the DMEC, TSC and Research Ethics Committee.

\section{Auditing}

The hospital Research and Development Department quality manager will conduct local monitoring of trial quality after the first patients have been enrolled. The trial database provides on-going data quality checking. The DMEC will look at recruitment rate, data quality and adverse event reporting and provide a summary report every 6 months.

\section{Ethics and dissemination}

\section{Research ethics approval}

The Greater Manchester East Research Ethics Committee granted ethical approval (REC reference: 13/NW/ 0782 61767) on the 19 November 2013. Informed patient consent will be obtained from all participants before enrolment in the trial.

\section{Protocol amendments}

Any significant protocol amendments will be communicated to the Research Ethics Committee and tabulated along with the amendment date and updated version number in the protocol. The local research team, the Trial Management Group, Trial Steering Committee and the Data Monitoring and Ethics Committees will all be informed.
The National Institute for Health Research (NIHR) portfolio team and the Controlled Trials Registry where the trial is registered will also be informed.

\section{Consent}

Please refer to the 'qualitative study', 'participant timeline', and 'recruitment' sections above for details on consent.

\section{Confidentiality}

Please refer to the 'data management' section above for details on confidentiality.

\section{Declaration of interests}

None.

\section{Access to data}

The trial statistician and the DMEC will have access to the final trial dataset.

\section{End of trial}

This will occur when the final patient has completed their 30-day post-operative follow-up and the final qualitative interview has taken place. At this point, all electronic and paper trial data will be securely archived for a minimum of 5 years in accordance with ICH GCP guidelines. Arrangements for confidential destruction of all documents will then be made. The trial may be stopped early upon recommendation of either the TSC or DMEC. If this occurs, all randomised patients will continue to be followed up as per the trial protocol.

\section{Post-trial care}

Due to the short duration of the intervention no posttrial care is required.

\section{Dissemination policy}

Following completion of the trial a report will be produced for the NIHR who have funded the trial. Several peer reviewed journal publications will be produced reporting various aspects of the trial and results will be presented at relevant scientific meetings. A lay version of the results will be available for all trial participants who request a copy, and for all hospital staff.

\section{Discussion}

Epidurals are in routine use in the National Health Service (NHS) [2,18], and their risks and benefits are well described; therefore this study does not confer any additional risk to trial patients. RSC carry a theoretical risk of needle damage to viscera on insertion (very unlikely under ultrasound guidance) and local anaesthetic toxicity (this applies to both interventions). However, to date no cases of either have been reported [23]. Clinical experience suggests good 
analgesia and easy mobility with RSC $[38,40]$ but the trial is required to provide direct comparison with epidurals.

We considered whether to blind the main study to minimise any potential bias. Unfortunately, blinding of staff would be impossible, as they are responsible for administering the drugs (by very distinct routes, with different pumps and different settings), as well as monitoring for adverse events related to the techniques. Single blinding (patient) was then considered but this was rejected for two reasons. These were the high likelihood of accidental unblinding, and the unacceptability of inserting sham epidurals or RSC. Allocation concealment will avoid selection bias but observer bias remains a risk. Blinding the data analyst to the intervention group will ameliorate this.

As we will be studying three different categories of surgery, with different expected rates of morbidity and hospital stay (secondary outcome measures), we have stratified randomisation to account for this and ensure these categories are balanced. The same applies to age. The vast majority of cancer cases are older-aged, however a younger cohort of patients with inflammatory bowel disease exists, for whom providing pain relief is notoriously difficult and hospital stays are often longer.

VAS pain scores are a necessary primary outcome measure for pain studies as they allow quantitative statistics. They are efficient to collect and easy for patients to understand, but are recognised as subjective in nature with individual variation, error in administration and limited to measuring pain intensity. Therefore, we will also be comparing a host of other measurements of quality of analgesia: for example, time to first rescue opiate, the amount of additional opiate, measurements of functional mobility and breathing, overall satisfaction with pain relief, measures of recovery and patient experience with their intervention. Several important secondary outcomes will allow further differentiation between the interventions regarding safety, experience and functional recovery.

There are several unique pragmatic aspects to the study design which will help to reduce confounders and improve the generalisability of results. Firstly, patients will have all other aspects of care standardised by inclusion in the ERP, with the respective trial interventions the only difference in processes of care. It is important to note that the respective trial interventions are not merely the insertion of an epidural compared to the insertion of a RSC, but rather two overall packages of analgesia and the way in which the interventions are managed: for example, the use of transdermal fentanyl patches in both groups at different appropriate time points, the universal use of gabapentin and simple analgesia, the portable pump system delivering the local anaesthetic via the RSC, robust protocols to optimise inadequate epidural or rectus sheath blocks and any breakthrough pain, and patient education pre-operatively regarding their allocated intervention, their role in their recovery and diary completion. A clear protocol states when an intervention is considered to have failed and the conversion to rescue analgesia.

Secondly, the use of transdermal fentanyl patches deserves further mention. These are used routinely in our institution as a step down from epidural analgesia. In order to manage the visceral pain component in the RSC arm, the patches are applied, following a single dose of morphine and a further RSC bolus, in the final 45 minutes of surgery. Visceral pain, although severe, is short-lived (6 to 24 hours) [23] and in our experience is effectively managed with a $12 \mathrm{mcg}$ or $25 \mathrm{mcg}$ patch. Previous studies have used morphine PCA in addition to the RSC for this visceral pain. However, there is a danger that the RSC are not optimised in these cases, with morphine being relied upon for any breakthrough pain. This would negate the benefits of a potentially good block, and could result in the side effects of high dose opiates as previously discussed. A morphine PCA would also require an additional pump and would further complicate mobilisation. Instead, we make use of oral morphine for any opiate required in addition to the patch.

Aside from increasing the quality of care provided to all patients participating in this study, further benefits to our institution include acquisition of new clinical skills for anaesthetic and ward staff regarding the insertion and management of RSC, new research skills and development of a research ethos for all staff involved, and potential reduction in morbidity and consequently hospital stay, leading to economic benefit.

Many surgical procedures that have previously been performed via midline incision are now being performed laparoscopically [56] or via transverse incisions. Neither of these approaches requires the interventions described in this trial. However, emergency laparotomies will continue to require an open midline approach. This group has a mortality rate 5 - to 10 -fold greater than the elective population, comprising in excess of 5,000 cases per year due to bowel cancer alone in England [56]. These vulnerable patients are frequently managed with high dose systemic opiate as many have contraindications to epidural analgesia. Therefore, if RSC demonstrate benefit, the technique may have widespread application in this surgical cohort.

\section{Trial status}

Recruitment began on the $10^{\text {th }}$ February 2014 with planned completion on the $10^{\text {th }}$ February 2016.

\section{Additional file}

Additional file 1: Rectus sheath catheter insertion technique. 


\section{Abbreviations}

AE: adverse event; ASA: American Society of Anesthesiologists score; Cl: chief investigator; CTU: Clinical Trials Unit; eCRF: electronic Case Report Form; DMEC: Data Management and Ethics Committee; ERP: Enhanced Recovery Programme; GCP: Good Clinical Practice; Gl: gastrointestinal; MAC: minimum alveolar concentration; NHS: National Health Service: NIHR: National Institute for Health Research; PCA: patient controlled analgesia; POAC: Pre-operative Assessment Clinic; POMS: Post-Operative Morbidity Survey; p-POSSUM: Physiologic and Operative Severity Score for the enUmeration of Mortality and morbidity; PQRS: Post-operative Quality Recovery Scale; R\&D: research and development; RBH: Royal Blackburn Hospital; RCT: randomised controlled trial; RfPB: Research for Patient Benefit programme; RSC: rectus sheath catheters; SAE: serious adverse event; TEA: thoracic epidural analgesia; TERSC: Thoracic Epidural analgesia versus Rectus Sheath Catheter study; TSC: Trial Steering Committee; VAS: Visual Analogue Scale.

\section{Competing interests}

The authors declare that they have no competing interests.

\section{Authors' contributions}

AK conceived of the study and led in its design and coordination. KMW drafted the manuscript and revised it following review by AK. SGB is the qualitative lead and is responsible for this section of the protocol. SL is the statistical lead and is responsible for these sections of the protocol. GC reviewed the background section and MS reviewed all sections of the manuscript. ACG reviewed the methodology section of the manuscript. All authors read and approved the final manuscript.

\section{Authors' information}

AK is a practicing Consultant in Anaesthesia and Intensive Care Medicine, Clinical Lead for all Enhanced Recovery Programmes for Surgery, Director of Research and Innovation for his institution, and Specialty Lead for Anaesthesia and Perioperative Medicine for the Greater Manchester Clinical Research Network as part of the National Institute for Health Research (NIHR), UK. ACG is Clinical Senior Lecturer in Intensive Care Medicine at Imperial College London and is an NIHR Clinician Scientist award holder. He is also Deputy Director of Research of the Intensive Care Foundation.

SGB is a lecturer with research interests which include patients' experiences of cancer (symptoms, care and services; using qualitative approaches to gather experiential data, and RCTs), physical and practical problems experienced by cancer survivors and the development, validation and use of patient reported outcome measures (PROMs).

\section{Acknowledgements}

The direct research costs of this study are being funded by a National Institute for Health Research (NIHR) Research for Patient Benefit Programme (RfPB) grant. As the study has been adopted onto the NIHR Research Portfolio (UKCRN ID 15847) it also benefits from all NHS service support costs. NHS employed researchers will be covered for negligent harm through the NHS CNORIS indemnity scheme.

This article presents independent research funded by the NIHR under its RfPB Programme (Grant Reference Number PB-PG-0212-27122). The views expressed are those of the authors and not necessarily those of the NHS, the $\mathrm{NIHR}$ or the Department of Health.

\section{Trial sponsor}

East Lancashire Hospitals NHS Trust. R\&D Ref: 2013/038.

\section{Author details \\ 'Department of Anaesthesia and Critical Care, Royal Blackburn Hospital, Blackburn BB2 3HH, UK. ${ }^{2}$ Faculty of Health and Medicine, Division of Health Research, Lancaster University, Lancaster LA1 4YT, UK. ${ }^{3}$ Department of Biostatistics, Duncan Building, University of Liverpool, Liverpool L69 3GA, UK. ${ }^{4}$ Department of Anaesthesia and Intensive Care, Royal Surrey County Hospital, University of Surrey, Guildford GU2 7XX, UK. ${ }^{5}$ Section of Anaesthetics, Pain Medicine and Intensive Care, Department of Surgery and Cancer, Imperial College, London SW7 2AZ, UK. ${ }^{6}$ Department of Surgery, Salford Royal NHS Foundation Trust, Stott Lane, Salford M6 8HD, UK.}

Received: 9 June 2014 Accepted: 3 October 2014

Published: 21 October 2014
References

1. Gustafsson UO, Scott MJ, Schwenk W, Demartines N, Roulin D, Francis N, McNaught CE, Macfie J, Liberman AS, Soop M, Hill A, Kennedy RH, Lobo DN, Fearon $\mathrm{K}$, Ljungqvist $\mathrm{O}$ : Guidelines for perioperative care in elective colonic surgery: Enhanced Recovery After Surgery (ERAS) Society Recommendations. World J Surg 2013, 37:259-284.

2. Cook TM, Eaton JM, Goodwin AP: Epidural analgesia following upper abdominal surgery: United Kingdom practice. Acta Anaesthesiol Scand 1997, 41:18-24.

3. Wheatley RG, Schug SA, Watson D: Safety and efficacy of postoperative epidural analgesia. Br J Anaesth 2001, 87:47-61.

4. Rigg JR, Jamrozik K, Myles PS, Silbert BS, Peyton PJ, Parsons RW, Collins KS: Epidural anaesthesia and analgesia and outcome of major surgery: a randomised trial. Lancet 2002, 359(9314):1276-1282.

5. Peyton PJ, Myles PS, Silbert BS, Rigg JA, Jamrozik K, Parsons R: Perioperative epidural analgesia and outcome after major abdominal surgery in high-risk patients. Anesth Analg 2003, 96(2):548.

6. Block BM, Liu SS, Rowlingson AJ, Cowan AR, Cowan JA Jr, Wu CL: Efficacy of postoperative epidural analgesia: a meta-analysis. JAMA 2003, 290(18):2455-2463

7. Werawatganon T, Charuluxananan S: Patient controlled intravenous opioid analgesia versus continuous epidural analgesia for pain after intraabdominal surgery. Cochrane Database Syst Rev 2005. Issue 1. Art. No. CD004088. doi:10.1002/14651858.CD004088.pub2.

8. Bonnet $F$, Marret $E$ : Influence of anaesthetic and analgesic techniques on outcome after surgery. Br J Anaesth 2005, 95(1):52-58.

9. Wood JD, Galligan JJ: Function of opioids in the enteric nervous system. Neurogastroenterol Motil 2004, 16(Suppl 2):17-28.

10. Behm B, Stollman N: Postoperative ileus: etiologies and interventions. Clin Gastroenterol Hepatol 2003, 1(2):71-80.

11. Wells C, Tinckler L, Rawlinson K, Jones H, Saunders J: Postoperative gastrointestinal motility. Lancet 1964, 1(7323):4-10.

12. Liu SS, Carpenter RL, Mackey DC, Thirlby RC, Rupp SM, Shine TS, Feinglass NG, Metzger PP, Fulmer JT, Smith SL: Effects of perioperative analgesic technique on rate of recovery after colon surgery. Anesthesiology 1995 83(4):757-765.

13. Steinbrook RA: Epidural anesthesia and gastrointestinal motility. Anesth Analg 1998, 86(4):837-844.

14. Jørgensen H, Wetterslev J, Møiniche S, Dahl JB: Epidural local anaesthetics versus opioid-based analgesic regimens for postoperative gastrointestinal paralysis, PONV and pain after abdominal surgery. Cochrane Database Syst Rev 2001. Issue 1. Art. No.: CD001893. doi:10.1002/14651858.CD001893.

15. Nakayoshi T, Kawasaki N, Suzuki Y, Urashima M, Hanyu N, Yanaga K: Epidural analgesia and gastrointestinal motility after open abdominal surgery - a review. J Smooth Muscle Res 2008, 44(2):57-64.

16. Morimoto H, Cullen JJ, Messick JM Jr, Kelly KA: Epidural analgesia shortens postoperative ileus after ileal pouch-anal canal anastomosis. Am J Surg 1995, 169(1):79-82.

17. Scott AM, Starling JR, Ruscher AE, DeLessio ST, Harms BA: Thoracic versus lumbar epidural anesthesia's effect on pain control and ileus resolution after restorative proctocolectomy. Surgery 1996, 120(4):688-695.

18. Cook TM, Counsel D, Wildsmith JAW: Major complications of central neuraxial block: report on the 3rd National Audit Project of the Royal College of Anaesthetists. Br J Anaesth 2009, 102:179-190.

19. Gould TH, Grace K, Thorne G, Thomas M: Effect of thoracic epidura anaesthesia on colonic blood flow. Br J Anaesth 2002, 89:446-451.

20. Ulf O, Gustafsson MD, Hausel J, Thorell A, Ljungqvist O, Soop M, Nygren J, for the Enhanced Recovery After Surgery Study Group: Adherence to the enhanced recovery after surgery protocol and outcomes after colorectal cancer surgery. Arch Surg 2011, 146(5):571-577.

21. Motamed C, Farhat F, Remerand F, Stéphanazzi J, Laplanche A, Jayr C: An analysis of postoperative epidural analgesia failure by computed tomography epidurography. Anaesthesia 2006, 103(4):1026-1032.

22. Krige A, Kelly L, Bannard-Smith J: Epidural failure and a rescue protocol: influence on hospital stay in colorectal surgery. Anaesthesia 2011, 66(Suppl 1):56

23. Webster $\mathrm{K}$ : Ultrasound guided rectus sheath block - analgesia for abdominal surgery. Update Anaesth 2010, 26:12-17.

24. Sandeman DJ, Dilley AV: Ultrasound-guided rectus sheath block and catheter placement. ANZ J Surg 2008, 78:621-623. 
25. Marhofer $\mathrm{P}$, Greher $M$, Kapral S: Ultrasound guidance in regional anaesthesia. Br J Anaesth 2005, 94:7-17.

26. Willschke H, Bosenberg A, Marhofer P, Johnston S, Kettner SC, Wanzel O, Kapral S: Ultrasonography-guided rectus sheath block in paediatric anaesthesia - a new approach to an old technique. Br J Anaesth 2006, 97(2):244-249.

27. Ferguson $S$, Thomas $V$, Lewis I: The rectus sheath block in paediatric anaesthesia: new indications for an old technique? Paediatr Anaesth 1996, 6(6):463-466.

28. Smith BE, Suchak M, Siggins D, Challands J: Rectus sheath block for diagnostic laparoscopy. Anaesthesia 1988, 43(11):947-948.

29. Dolan J, Lucie P, Geary T, Smith M, Kenny G: The rectus sheath block for laparoscopic surgery in adults: a comparison between the loss of resistance and ultrasound guided techniques. Anaesthesia 2007, 62:302.

30. Wada M, Kitayama M, Hashimoto H, Kudo T, Takada N, Hirota K: Plasma ropivacaine concentrations after ultrasound-guided rectus sheath block in patients undergoing lower abdominal surgery. Anesth Analg 2012, 114(1):230-232.

31. Hessian EC, Evans BE, Woods JA, Taylor DJ, Kinkel E, Bjorksten AR: Plasma ropivacaine concentrations during bilateral transversus abdominis plane infusions. Br J Anaesth 2013, 111(3):488-495.

32. Petersen PL, Hilsted KL, Dahl JB, Mathiesen O: Bilateral transversus abdominis plane (TAP) block with 24 hours ropivacaine infusion via TAP catheters: a randomized trial in healthy volunteers. BMC Anesthesiol 2013, $13: 30$

33. Gill AM, Scott NB, Abbas M: Ropivacaine plasma levels following local infiltration analgesia for primary total hip arthroplasty. Anaesthesia 2014 69(4):368-373

34. Watson D, Farquhar XX, Dennison A, Cole J, McAllister S: Postoperative analgesia by infusion of local anaesthetic into the rectus sheath. Br J Anaesth 1991, 67:656.

35. Padmanabhan J, Rohatgi A, Niaz A, Chojnowska E, Baig K, Woods WGA: Does rectus sheath infusion of bupivacaine reduce postoperative opioid requirement? Ann $R$ Coll Surg Engl 2007, 89:229-232.

36. Cornish $P$, Deacon $A$ : Rectus sheath catheters for continuous analgesia after upper abdominal surgery. ANZ J Surg 2007, 77(12):84

37. Shido A, Imamachi N, Doi K, Sakura S, Saito Y: Continuous local anesthetic infusion through ultrasound-guided rectus sheath catheters. Can J Anaesth 2010, 57(11):1046-1047.

38. Parsons B, Aning J, Daugherty M, McGrath JS: The use of rectus sheath catheters for patients undergoing radical cystectomy. BJMSU 2011 4:24-30.

39. Khorgami Z, Shoar S, Hosseini Araghi N, Mollahosseini F, Nasiri S, Ghaffari $\mathrm{MH}$, Aminian A: Randomized clinical trial of subcutaneous versus interfascial bupivacaine for pain control after midline laparotomy. BJS 2013, 100(6):743-748

40. Dutton TJ, McGrath JS, Daugherty MO: Use of rectus sheath catheters for pain relief in patients undergoing major pelvic urological surgery. BJ Int 2014, 113:246-253.

41. Morse J, Stern P, Corbin J, Bowers B, Clarke A, Charmaz K: Developing Grounded Theory: The Second Generation (Developing Qualitative Inquiry). Walnut Creek: Left Coast Press; 2009

42. McCormack HM, Horne DJ, Sheather S: Clinical applications of visual analogue scales: a critical review. Psychol Med 1988, 18:1007-1019.

43. Huskisson EC: Measurement of pain. Lancet 1974, 2:1127-1131.

44. Royse CF, Newman S, Chung F, Stygall J, McKay RE, Boldt J, Servin FS, Hurtado I, Hannallah R, Yu B, Wilkinson DJ: Development and feasibility of a scale to assess postoperative recovery: the post-operative quality recovery scale. Anaesthesiology 2010, 113(4):892-905.

45. Fiore J Jr, Browning P, Bialocerkowski A, Gruen RL, Faragher IG, Denehy L: Hospital discharge criteria following colorectal surgery: a systematic review. Colorectal Dis 2012, 14(3):270-281.

46. Krige A, Kelly L, Bannard-Smith J: Surgical approach, analgesic techniques and length of stay in colorectal surgery. Anaesth 2011, 66(Suppl 1):56.

47. Ludwig K, Enker WE, Delaney CP, Wolff BG, Du W, Fort JG, Cherubini M, Cucinotta J, Techner L: Gastrointestinal tract recovery in patients undergoing bowel surgery. Arch Surg 2008, 143(11):1098-1105.

48. Grocott M, Browneb J, Van der Meulen J, Matejowsky C, Mutch M, Hamilton MA, Levett DZ, Emberton M, Haddad FS, Mythen MG: The postoperative morbidity survey was validated and used to describe morbidity after major surgery. J Clin Epidemiol 2007, 60:919-928.
49. Dindo D, Desmartines N, Clavien PA: Classification of surgical complications. Ann Surg 2004, 240:205-213.

50. Tilleul P, Aissou M, Bocquet F, Thiriat N, le Grelle O, Burke MJ, Hutton J, Beaussier M: Cost-effectiveness analysis comparing epidural, patientcontrolled intravenous morphine, and continuous wound infiltration for postoperative pain management after open abdominal surgery. Br J Anaesth 2012, 108(6):998-1005.

51. Kelly AM: The minimum clinically significant difference in visual analogue scale pain score does not differ with severity of pain. Emerg Med J 2001, 18:205-207.

52. American Society of Anesthesiologists: ASA Physical Status Classification System. [http://www.asahq.org/Home/For-Members/Clinical-Information/ ASA-Physical-Status-Classification-System]

53. Tekkis PP, Kessaris N, Kocher HM, Poloniecki JD, Lyttle J, Windsor AC: Evaluation of POSSUM and P-POSSUM scoring systems in patients undergoing colorectal surgery. Br J Surg 2003, 90(3):340-345.

54. Grant AM, Altman DG, Babiker AB, Campbell MK, Clemens FJ, Darbyshire JH, Elbourne DR, McLeer SK, Parmar MK, Pocock SJ, Spiegelhalter DJ, Sydes MR, Walker AE, Wallace SA, for the DAMOCLES study group: Issues in data monitoring and interim analysis of trials. Health Technol Assess 2005, 9(7):1-238.

55. DAMOCLES study group: A proposed charter for clinical trial data monitoring committees: helping them to do their job well. Lancet 2005, 365:711-722.

56. Scott N, Smith J, Hill J: National Bowel Cancer Audit Annual Report. 2013 [http://www.hscic.gov.uk/catalogue/PUB11105/nati-clin-audi-supp-progbowe-canc-2013-rep1.pdf]

\section{doi:10.1186/1745-6215-15-400}

Cite this article as: Wilkinson et al:: Thoracic Epidural analgesia versus Rectus Sheath Catheters for open midline incisions in major abdominal surgery within an enhanced recovery programme (TERSC): study protocol for a randomised controlled trial. Trials 2014 15:400.

\section{Submit your next manuscript to BioMed Central and take full advantage of:}

- Convenient online submission

- Thorough peer review

- No space constraints or color figure charges

- Immediate publication on acceptance

- Inclusion in PubMed, CAS, Scopus and Google Scholar

- Research which is freely available for redistribution 